\title{
Prehypertension in disease-free adults: a marker for an adverse cardiometabolic risk profile
}

\begin{abstract}
Alok K Gupta, Meghan McGlone, Frank L Greenway and William D Johnson
Cardiovascular disease (CVD) is the leading cause of death worldwide. Understandably, cardiometabolic risk assessment is an integral component of every adult health evaluation. Customary assessment measures are, however, inadequate: as two-thirds of sudden cardiac deaths occur in clinically healthy individuals. Novel indicators favoring early recognition of adverse cardiometabolic risk in disease-free adults are clearly needed. Clinically healthy disease-free adults with prehypertension (PreHTN: BP120-139/80-89 mm Hg) have an adverse cardiometabolic risk profile. A statistical analysis of disease-free adult NHANES participants was conducted from 1999 to 2006. Overall prevalence of PreHTN in disease-free adults was $36.3 \%$. Prevalence was higher in men $(P<0.001)$ increasing with age up to 70 years $(P<0.001)$. Prevalence correlated strongly with indicators of adverse cardiometabolic risk profile: it was higher with increasing body mass index (BMI) and waist circumference (WC) $(P<0.001$ for both). Means were significantly higher for BMI, WC, glucose, insulin, hemoglobin A1c, homeostasis model assessment, pulse pressure, C-reactive protein, total cholesterol and triglycerides in subjects with PreHTN (vs. desirable BP: $P<0.05$ for all). Prevalence of two or more unfavorable risk factors (other than high BP) was $30 \%$ higher in disease-free adults with PreHTN vs. desirable BP (prevalence ratio: 1.30; 95\% Cl: 1.22, 1.39). Detection of PreHTN (a precursor for subsequent HTN), during annual health maintenance in disease-free adults, (especially with one or more of the recognized CVD risk correlates), could become an early marker of adverse cardiometabolic risk profile. Clinical care designed to prevent progression from PreHTN to HTN (JNC 7 recommendation) may attenuate risk.
\end{abstract}

Hypertension Research (2010) 33, 905-910; doi:10.1038/hr.2010.91; published online 10 June 2010

Keywords: BMI; insulin resistance; waist circumference; CRP; metabolic syndrome

\section{INTRODUCTION}

During ages 40-69 years and even beyond, each 20/10 $\mathrm{mm} \mathrm{Hg}$ increment of blood pressure (BP) over the usual or desirable systolic blood pressure/diastolic blood pressure (SBP/DBP) of $120 / 80 \mathrm{~mm} \mathrm{Hg}$ is associated with about a twofold increase in death rate from cerebrovascular events (hemorrhage or ischemia), ischemic heart disease or other vascular causes. ${ }^{1}$ This twofold increase holds true for BP levels above $115 / 75 \mathrm{~mm} \mathrm{Hg} .{ }^{1}$ Each $5 \mathrm{~kg} / \mathrm{m}^{2}$ increment in body mass index (BMI) above an apparent optimum of $22.5-25 \mathrm{~kg} \mathrm{~m}^{-2}$, is associated with about a $30 \%$ higher overall risk for mortality (hazard ratio per $5 \mathrm{~kg} \mathrm{~m}^{-2}$ (HR) 1.29 (95\% confidence interval (95\% CI): 1.27-1.32)), primarily from vascular events, including diabetes mellitus. ${ }^{2}$ Enlarged waist circumference (WC), a strong correlate of BMI and a clinical marker for an expanding visceral adipose tissue compartment, has been associated with multiple untoward metabolic derangements that increase the likelihood of cardiovascular morbidity and mortality. ${ }^{3-5}$ Higher levels of serum triglycerides (TGs) (>150 mg per $100 \mathrm{ml}$ ) and low levels of high-density lipoprotein-cholesterol (HDL-C) $(<40 \mathrm{mg}$ per $100 \mathrm{ml}$ in men and $<50 \mathrm{mg}$ per $100 \mathrm{ml}$ in women) have been similarly associated with elevated risk for vascular events. ${ }^{6-8}$ Fasting serum glucose (FSG) levels over $100 \mathrm{mg}$ per $100 \mathrm{ml}$ increase the risk for developing cardiovascular disease (CVD). ${ }^{9}$ Prediabetes (American Diabetes Association criteria: fasting blood glucose $100-125 \mathrm{mg}$ per $100 \mathrm{ml}$ and/or $2 \mathrm{~h}$ post-meal blood glucose $140-199 \mathrm{mg}$ per $100 \mathrm{ml}$ ) is associated with abnormal circadian BP variability, ${ }^{10}$ a plausible functional measure of the mechanism underlying increased adverse cardiovascular events.

This has lead to the recognition of the metabolic syndrome, a cluster of abnormal clinical, and/or laboratory markers, associated with high cardiovascular morbidity and mortality. ${ }^{11}$ The World Health Organization, ${ }^{12}$ National Cholesterol Education Program, Adult Treatment Panel $\mathrm{III}^{13}$ and International Diabetes Federation ${ }^{14}$ have, over the years, proposed varying criteria for the diagnosis of the metabolic syndrome. The World Health Organization categorizes the five criteria into one related to glycemic parameters (which have changed after the initial description; American Diabetes Association diagnostic criteria for diabetes mellitus) and the other four that relate to WC, TG, HDL$\mathrm{C}$ and microalbuminuria. The Adult Treatment Panel III criteria relate to their focus on CVD risk and primary prevention. Presence of any three of the five criteria (fasting glucose $>110 \mathrm{mg}$ per $100 \mathrm{ml}$; WC $>102 \mathrm{~cm}$ in men or $>88 \mathrm{~cm}$ in women; TG $>150 \mathrm{mg}$ per $100 \mathrm{ml}$; HDL-C $<40 \mathrm{mg}$ per $100 \mathrm{ml}$ in men or $<50 \mathrm{mg}$ per $100 \mathrm{ml}$ in women; 
BP $>130 / 85 \mathrm{~mm} \mathrm{Hg}$ ) would be diagnostic of the metabolic syndrome. ${ }^{13}$ The International Diabetes Federation definition includes a fasting glucose $>100 \mathrm{mg}$ per $100 \mathrm{ml}$ (instead of $>110 \mathrm{mg}$ per $100 \mathrm{ml}$ ) and ethnic group-specific WC cutoffs. ${ }^{14}$

The revision of the BP categories by the Seventh Report of the Joint National Committee on Prevention, Detection, Evaluation and Treatment of High Blood Pressure (JNC 7) further compounded the ensuing confusion. ${ }^{15}$ The JNC 7 has placed the desirable BP below $120 / 80 \mathrm{~mm} \mathrm{Hg}$ and high BP (or hypertension) over 140/90 mm Hg. Hypertension has long been recognized as a high CVD risk state meriting drug therapy to lower the $\mathrm{BP}$ into the desirable range. Prehypertension (PreHTN), with a BP between 120-139/80$89 \mathrm{~mm} \mathrm{Hg}$, on the other hand, is not a well-studied condition. The CVD risk for PreHTN, perceived to be greater than that for desirable $\mathrm{BP}$, has not been quantified. Although PreHTN usually rapidly deteriorates into hypertension at a rather alarming rate, the CVD risk with PreHTN, as an independent entity, is unclear.

Although it is well recognized that frank unmedicated hypertension is associated with increased CVD risk, as well as an increased risk for the metabolic syndrome, the degree of risk for individuals with PreHTN remains debatable. We investigated the differences in otherwise healthy disease-free adults with PreHTN vs. normotension with respect to WC ( $>102 \mathrm{~cm}$ in men and $>88 \mathrm{~cm}$ in women), TGs $(>150 \mathrm{mg}$ per $100 \mathrm{ml})$, HDL-C $(<40 \mathrm{mg}$ per $100 \mathrm{ml}$ in men and $<50 \mathrm{mg}$ per $100 \mathrm{ml}$ in women) and FSG (>100 mg per $100 \mathrm{ml}$ ). These qualifiers, besides each having widespread recognition in the literature for independently elevating CVD risk in otherwise healthy subjects, ${ }^{3-10}$ are the components of the metabolic syndrome. ${ }^{16}$ In this cross-sectional survey of the population, evidence of a link between PreHTN and the metabolic syndrome also provides an indirect evidence of increased CVD risk. We tested the hypothesis that PreHTN in disease-free adults is reflective of a latent risk for adverse cardiometabolic outcomes, the early recognition of which could offer potential for JNC 7 recommended risk reduction.

\section{METHODS}

Study sample

Analyses were conducted using the data from the United States National Health and Nutrition Examination Survey (US NHANES), from 1999 to 2006. NHANES uses a complex, multistage, probability sampling design to select a representative sample of the noninstitutionalized, civilian US population. Sample weights assigned to each individual allow for the calculation of valid national prevalence estimates. ${ }^{17}$ The National Center for Health Statistics ethics review board approved the original survey protocols. An informed consent was obtained from all NHANES participants. Trained personnel conducted home interviews for reliable data collection. This included demographic, socioeconomic, dietary and health-related information. A mobile exam center was used to obtain anthropometric measurements and secure a fasting blood draw. Medical personnel were used to obtain medical, dental and physiological measurements, as well as the results from the laboratory tests. Details on these and other assessments can be accessed at the NHANES website. ${ }^{18}$

\section{Sample methods}

Participants were required to come to a mobile examination clinic before 0900 hours after fasting for at least $9 \mathrm{~h}$. If they arrived having fasted for $<8.5 \mathrm{~h}$, they received an analytical sampling weight equal to zero, as part of the NHANES protocol. Blood was drawn from an antecubital vein of the left arm. Fasting plasma glucose was assessed by the hexokinase method. TGs and HDL-C were also assayed from the fasting serum samples. Seated resting BP was measured after the participant had rested quietly for $5 \mathrm{~min}$. Three consecutive BP readings were obtained 1-min apart. The average of these was recorded as the resting BP. Weight was measured with participants wearing minimal clothing. Height was measured using a fixed wall stadiometer, heels together and arms by the side,
Table 1 Commonly used reference values for cardiovascular risk factors

\begin{tabular}{lc}
\hline Cardiometabolic risk category & Desirable range \\
\hline Waist circumference (male) & $<102 \mathrm{~cm}$ \\
Waist circumference (female) & $<88 \mathrm{~cm}$ \\
Glucose & $<100 \mathrm{mg}$ per $100 \mathrm{ml}$ \\
Pulse pressure & $<60 \mathrm{~mm} \mathrm{Hg}$ \\
Total cholesterol & $<200 \mathrm{mg}$ per $100 \mathrm{ml}$ \\
Triglycerides & $<150 \mathrm{mg}$ per $100 \mathrm{ml}$ \\
HDL cholesterol (male) & $>40 \mathrm{mg}$ per $100 \mathrm{ml}$ \\
HDL cholesterol (female) & $>50 \mathrm{mg}$ per $100 \mathrm{ml}$
\end{tabular}

Abbreviation: HDL, high-density lipoprotein.

with the eyes in the Frankfort plane. WC was measured at the end of normal expiration with the participant in a standing position using a steel measuring tape placed at the high point of the iliac crest, to the nearest $0.1 \mathrm{~cm}$. Race or ethnicity was derived from questions about race and Hispanic origin (nonHispanic white, Mexican American, non-Hispanic black and 'other').

\section{Diagnosis of PreHTN}

The JNC 7 criteria for PreHTN (SBP $120-139 \mathrm{mmHg}$ and/or DBP 80 $89 \mathrm{~mm} \mathrm{Hg}$, with neither $\mathrm{SBP} \geqslant 140 \mathrm{~mm} \mathrm{Hg}$ or $\mathrm{DBP} \geqslant 90 \mathrm{~mm} \mathrm{Hg}$ ) in adults not taking any medications for hypertension provided the diagnosis of PreHTN. ${ }^{15}$ We further defined Level 1 PreHTN as SBP $120-129 \mathrm{~mm} \mathrm{Hg}$ and/ or DBP $80-84 \mathrm{~mm} \mathrm{Hg}$ with neither SBP $\geqslant 130 \mathrm{~mm} \mathrm{Hg}$ or DBP $\geqslant 85 \mathrm{~mm} \mathrm{Hg}$ : and Level 2 PreHTN as SBP $130-139 \mathrm{~mm} \mathrm{Hg}$ and/or DBP $85-89 \mathrm{~mm} \mathrm{Hg}$ with neither $\mathrm{SBP} \geqslant 140 \mathrm{~mm} \mathrm{Hg}$ or DBP $\geqslant 90 \mathrm{~mm} \mathrm{Hg}$.

\section{Unmedicated hypertension}

Participants were defined as having unmedicated hypertension if on screening their SBP was $\geqslant 140 \mathrm{~mm} \mathrm{Hg}$ and/or DBP was $\geqslant 90 \mathrm{~mm} \mathrm{Hg}$ and they selfreported not taking any medication for treatment of high BP.

\section{Cardiometabolic profile}

Reference levels for desirable WC (for men $<102 \mathrm{~cm}$ and for women $<88 \mathrm{~cm}$ ), glucose $(<100 \mathrm{mg}$ per $100 \mathrm{ml})$, pulse pressure $(<60 \mathrm{~mm} \mathrm{Hg})$, total cholesterol (total-C $<200 \mathrm{mg}$ per $100 \mathrm{ml}$ ), TG ( $<150 \mathrm{mg}$ per $100 \mathrm{ml}$ ) and HDL-C (in men $>40 \mathrm{mg}$ per $100 \mathrm{ml}$, and in women $>50 \mathrm{mg}$ per $100 \mathrm{ml}$ ) are detailed in Table 1 .

\section{Statistical analysis}

Statistical analyses were performed using procedures for weighted sample survey data available in SAS software (SAS Version 9.1, Cary, NC, USA). Level of a condition was reported as a mean (standard error). Cardiometabolic risk factors were each summarized as a percentage (standard error). Significance of the difference between two summary estimates was reported in terms of a $P$-value based on large sample $z$-tests for the null hypothesis of equality $v s$. two-directional alternatives. A prevalence ratio and its corresponding $95 \%$ CI were calculated to assess the prevalence of having two or more disordered CVD risk factors in adults who had PreHTN relative to those who had normotension.

\section{RESULTS}

\section{Analytical sample}

The 1999-2006 NHANES samples included 41474 participants. This study represents 20311 adults over the age of 20 years (21 163 participants that were under 20 years of age were excluded). The female participants with an ongoing pregnancy (1169) were also excluded. Other exclusions were 1253 for partaking in the interview portion only; 1813 for not having had BP, BMI or WC measurements; and 3891 for having a history of coronary heart disease, congestive heart failure, angina, myocardial infarction, stroke or cancer. Finally, 


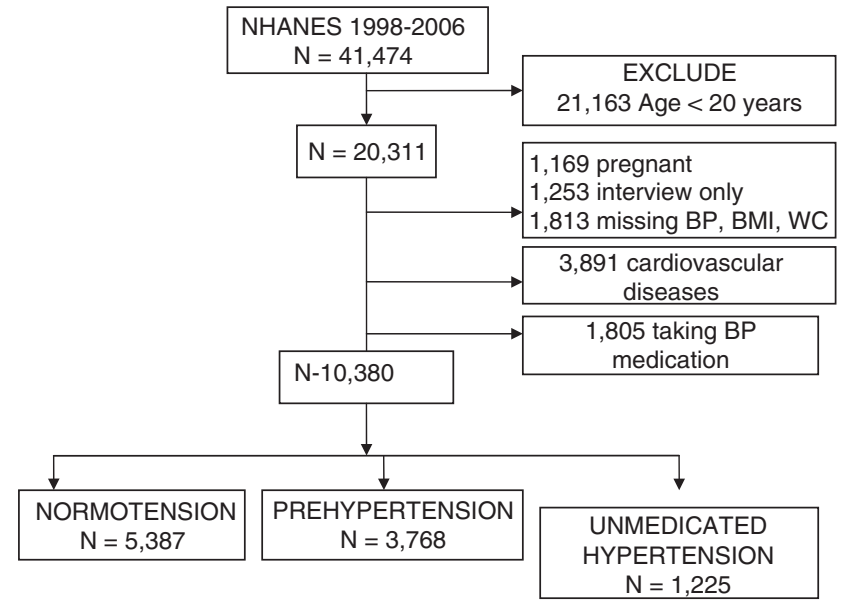

Figure 1 Schema for data inclusion into the analytical sample.

an additional 1805 were not considered for reportedly taking prescription medication/s for high BP (9931 excluded from 20311). Thus with 31094 of 41474 participants not meeting the inclusion criteria, a final sample of disease-free healthy adults for this report was 10380 (Figure 1).

\section{Prevalence of PreHTN}

The overall prevalence of normal BP, PreHTN and unmedicated hypertension in the healthy disease-free US adult population was $51.9(0.9) \%, 36.3(0.7) \%$ and $11.8(0.6) \%$, respectively (Table 2$)$. Men had a higher prevalence of PreHTN compared to women (44.8 (0.9)\% vs. $27.3(0.9) \%)$; prevalence increased with age (31.2 (0.9)\% for ages 20-39 years, 42.3 (1.0)\% for ages $40-59$ years and $44.2(2.0) \%$ for ages 60-69 years). Mexican Americans had a significantly lower prevalence (32.2 (1.0)\%), compared to non-Hispanic white $(36.9(0.9) \%)$ and non-Hispanic black (38.9 (1.2)\%). Prevalence of PreHTN varied only slightly with smoking status $(35.9$ (0.9)\%, 37.7 (1.3)\%, $36.0(1.0) \%$ prevalence in non-, former and current smokers, respectively). Prevalence was not significantly different between nondrinkers and those who consumed 1-4 alcoholic drinks per day (35.4 (0.9)\%), but it was significantly higher in those who consumed $\geqslant 5$ drinks per day $(42.7(1.6) \%)$.

The prevalence of PreHTN was significantly higher in men with WC $\geqslant 102 \mathrm{~cm}$ compared to those with WC $<102 \mathrm{~cm}$ (49.3 vs. $42.6 \%$, $P<0.001)$. Similarly, prevalence was significantly higher in women with WC $\geqslant 88 \mathrm{~cm}$ compared to those with WC $<88 \mathrm{~cm}(33.9$ vs. $20.4 \%, P<0.001)$. This higher prevalence of PreHTN associated with large WC (in age groups below 60 years) was observed for women in all ethnicities and for men in all ethnicities except non-Hispanic black and other ethnicities.

A graduated increase in overall prevalence of PreHTN was seen with an increasing BMI: lean (BMI $\left.<18.5 \mathrm{~kg} \mathrm{~m}^{-2}\right) 18.7 \pm 3.3 \%$, normal weight (BMI 18.5-24.9 $\mathrm{kg} \mathrm{m}^{-2}$ ) 30.6 (1.0)\%, overweight (BMI 25$\left.30 \mathrm{~kg} \mathrm{~m}^{-2}\right) 38.0(0.8) \%$ and obese (BMI $\left.>30 \mathrm{~kg} \mathrm{~m}^{-2}\right) 43.1(1.2) \%$ $(P<0.001$ for trend). This is graphically illustrated in Figure 2. Men exhibited an increase across normal weight, overweight and obese categories: $40.7(1.6) \%, 45.2(1.2) \%$ and $50.5(1.5) \%$, respectively. Women mirrored these increases at 22.3 (1.2)\%, 26.9 (1.6)\% and 36.0 (1.8)\%. Congruent increases were seen with ages $20-39$ years $(24.7$ (1.5)\%, 34.8 (1.4)\%, 39.4 (1.4)\%), 40-59 years (38.1 (1.7)\%, 41.0 (1.4)\%, $48.7(2.1) \%$ ) and 60-69 years (43.1 (3.7)\%, $47.2(2.8) \%, 41.4$ $(3.2) \%)$. All ethnic categories had an increase in prevalence of
PreHTN (and concurrent decrease in normotension) with increasing BMI, with Mexican Americans showing the lowest rates.

The overall prevalence of normotension and PreHTN, respectively, are also shown to decrease and increase incrementally as WC increases in both men and women $(P<0.001)$. This is graphically illustrated in Figure 2. Analogous significant increases were observed in men and women as shown, in the 20-39, 40-59 and 60-69 year age groups, and in non-Hispanic white, non-Hispanic black and Mexican Americans.

Unmedicated hypertension was higher in men compared to women, drastically higher in the elderly, higher in non-Hispanic black and lower in Mexican Americans, higher in former smokers but lower in current smokers and higher in nondrinkers of alcohol (Figure 3).

\section{Cardiometabolic risk profile}

Excluding BP, $40 \%$ of the participants in our analytical sample had two or more criteria for the diagnosis for the metabolic syndrome; two or more criteria were found in 32.1, 46.1 (43.7\% in Level 1 and $49.95 \%$ in Level 2 PreHTN) and 54.6 of disease-free healthy subjects with normotension, PreHTN and unmedicated hypertension, respectively.

Summary statistics for individual cardiometabolic risk factors are shown in Table 3. Mean BMI was significantly higher in subjects with PreHTN compared to those with desirable BP (28.3 vs. 26.3, $P<0.001)$ and in untreated subjects with hypertension compared to those with PreHTN (29.1 vs. 28.3, $P<0.01)$. Similar significant incremental increases among subjects with normotension, PreHTN and unmedicated hypertension (indicative of higher cardiometabolic risk) were observed for WC (men and women), glucose, insulin, hemoglobin Alc, homeostasis model assessment, pulse pressure, Creactive protein, total-C, low-density lipoprotein-cholesterol (LDL-C), TG and total-C/HDL-C ratio. In women, the LDL-C/HDL-C ratio was significantly higher in subjects with PreHTN compared to those with desirable BP. The prevalence ratio for having two or more risk factors (other than high BP) outside of the normal range, in adults with PreHTN vs. subjects with desirable BP, was 1.30 (95\% CI: 1.22, 1.39), indicating 30\% higher prevalence with PreHTN.

Table 4 details the number of subjects with normotension, PreHTN and unmedicated hypertension with $<2$ or $>2(0$ or 1 and 2,3 or 4$)$ cardiometabolic risk factors.

\section{DISCUSSION}

The 1999-2006 NHANES survey indicated that nearly 50\% of clinically healthy and apparently disease-free US adults have either PreHTN or unmedicated hypertension; an estimated $36.3 \%$ have BP levels indicative of PreHTN and $11.8 \%$ have unmedicated hypertension. The prevalence of PreHTN increases not only with age and BMI, but also with larger WC, increased TGs and FSG. All these (larger WC, increased TGs and FSG, along with lower HDL-C levels), in addition to being the components and the criteria for the diagnosis for the metabolic syndrome, ${ }^{11}$ are also well-established markers for increased CVD risk. ${ }^{3-9}$ The prevalence of two or more disordered cardiometabolic risk factors other than high BP was significantly higher in adults with PreHTN. The fact that subjects with hypertension significantly differ from those with desirable BP (normotension) in BMI, WC, FSG, hemoglobin A1c, total-C, LDL-C, whereas the subjects with PreHTN differ significantly from those with normotension in BMI, WC, FSG, hemoglobin A1c, Insulin, homeostasis model assessment, C-reactive protein, total-C, LDL-C, TG and cardiac risk ratios attests to PreHTN being strongly associated with an adverse cardiometabolic risk profile.

There has been much debate over the CVD risk associated with PreHTN (BP 120-139/80-89 mm Hg). An increase in CVD risk is 
Table 2 Estimated weighted prevalence (s.e.) of normal blood pressure, prehypertension and unmedicated hypertension in disease-free adults

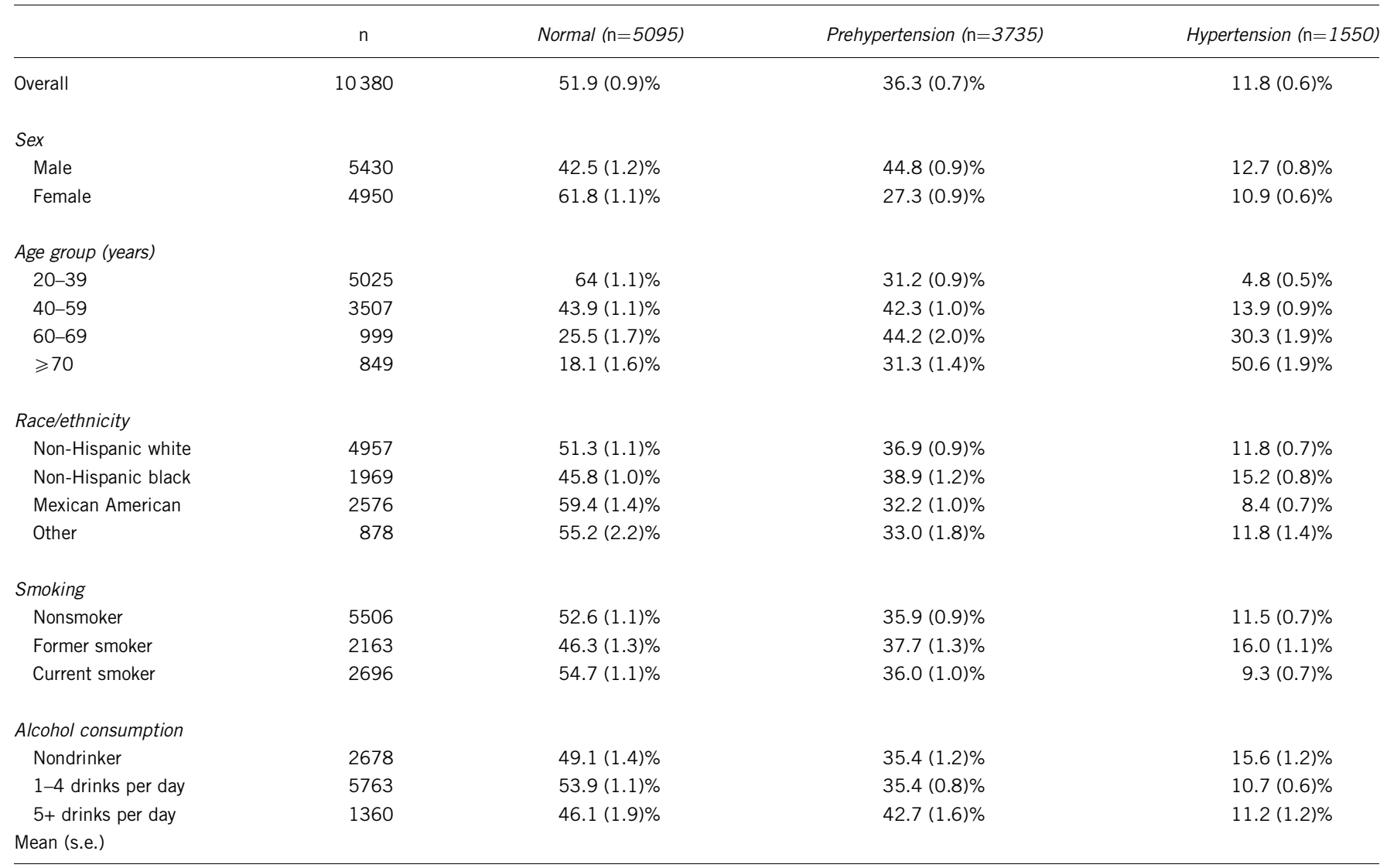

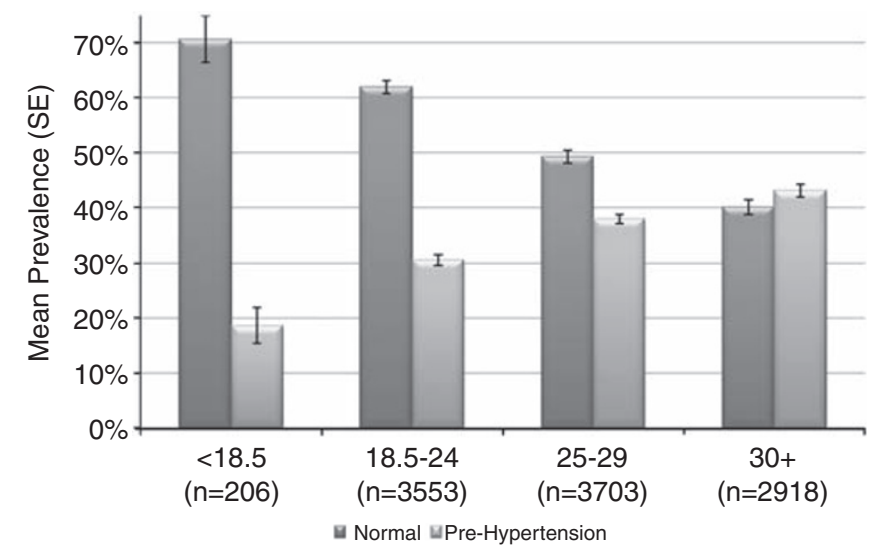

Figure 2 Estimated weighted prevalence (s.e.) of normal blood pressure and prehypertension in the disease-free US adult population by BMI.

found on either side of a BP threshold of $115 / 75 \mathrm{~mm} \mathrm{Hg} .{ }^{19}$ A very high proportion of subjects (37.3\%) with Level 1 PreHTN or high normal BP (JNC 6: BP 130-139/85-89 mm Hg) deteriorate into hypertension in 4 years in the Framingham cohort. ${ }^{20} \mathrm{~A}$ similar proportion of disease-free subjects (38\%) with PreHTN (JNC 7: BP 120-139/80$89 \mathrm{~mm} \mathrm{Hg}$ ) progressed to hypertension over a 4-year period in the Strong Heart Study. ${ }^{21}$ Both studies represent diverse cohorts (Framingham - a sample from of Framingham city in Massachusetts, and the Strong Heart Study-a sample of American Indians) and use different criteria for classification (high normal BP, JNC 6 and

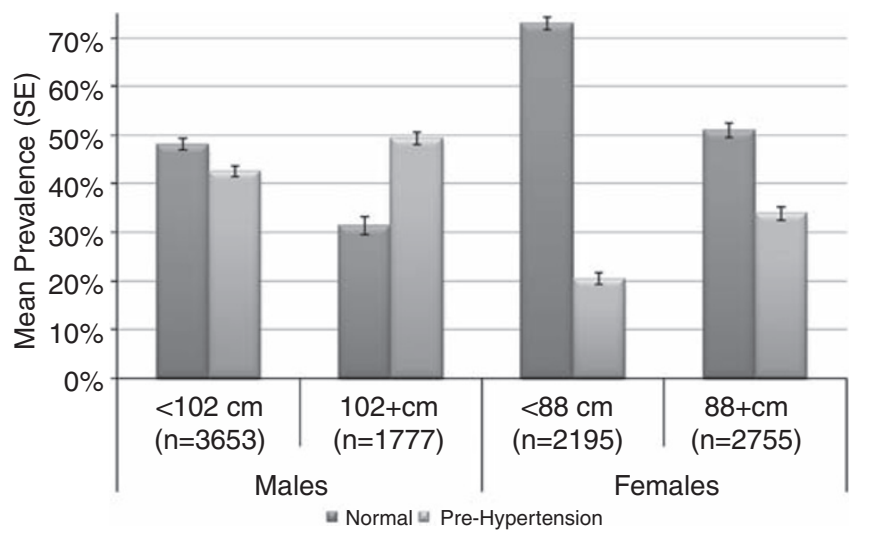

Figure 3 Estimated weighted prevalence (s.e.) of normal blood pressure and prehypertension in the disease-free US adult population by waist circumference.

PreHTN, JNC 7 criteria, respectively). Subjects in both these studies, however, deteriorated over 4 years at almost identical rates (37.3 and $38 \%)$ into hypertension. Relative risk for PreHTN when compared with normal BP was higher (all-cause HR 1.27, 95\% CI: 1.02-1.58); CVD mortality HR 1.66, 95\% CI: 1.21-2.26) in NHANES II (19761980). ${ }^{22}$ These differences were, however, negated when adjustments for CVD risk factors were instituted. ${ }^{22}$ In a subsequent study with NHANES III (follow-up through 2000) compared with normotension, the relative risks of CVD mortality for PreHTN were 1.23 (95\% CI: $0.85-1.79, P=0.26) .^{23}$ 
Table 3 Mean (s.e.) of cardiovascular risk factors in disease-free adults with normal blood pressure, prehypertension and un-medicated hypertension and $\boldsymbol{P}$-values of testing between groups

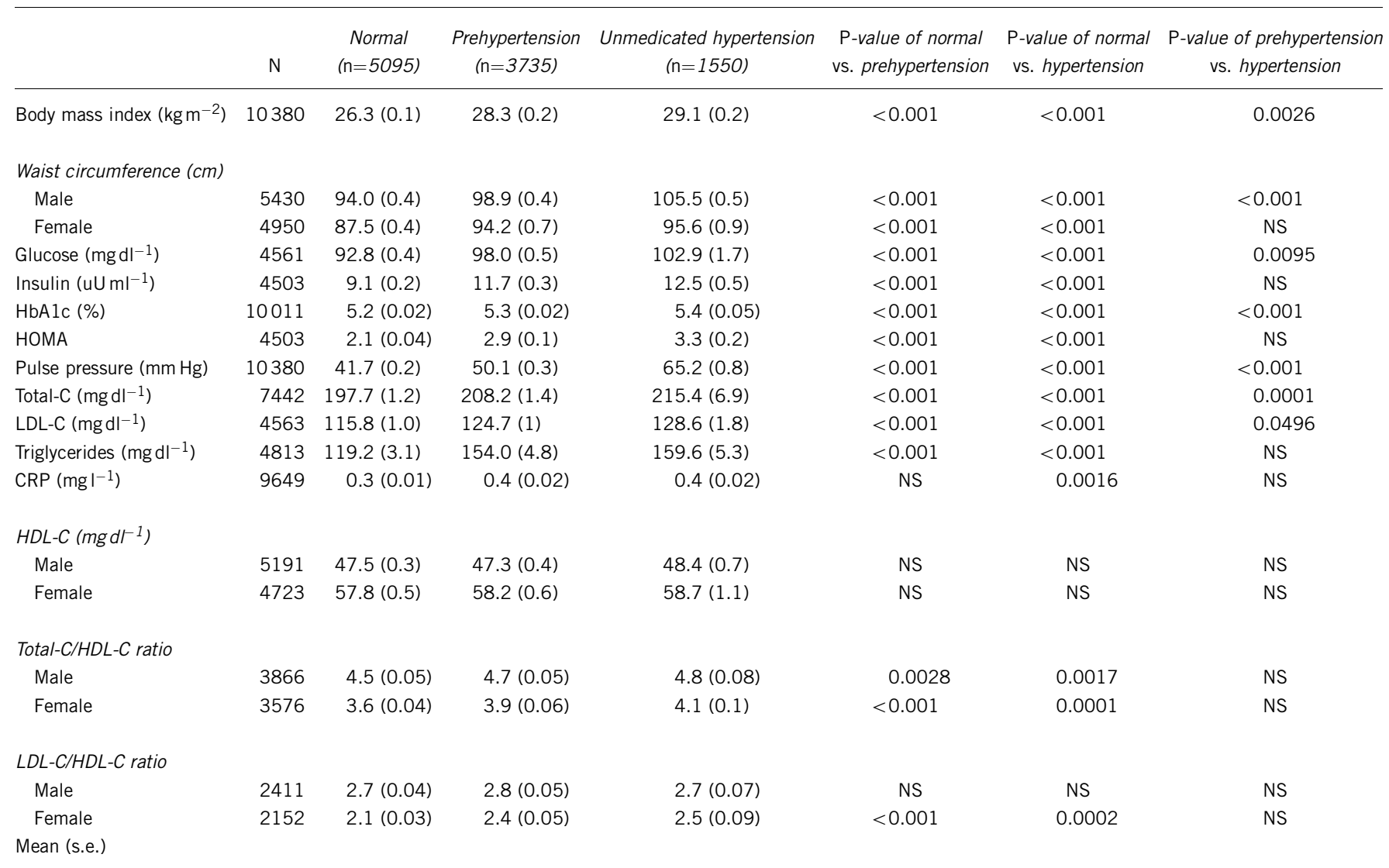

Abbreviations: CRP, C-reactive protein; Hb1Ac, hemoglobin A1c; HDL-C, high-density lipoprotein-cholesterol; HOMA, homeostasis model assessment; LDL-C, low-density lipoprotein-cholesterol; total-C, total cholesterol.

Table 4 Cardiometabolic risk factors in normal blood pressure, prehypertension and unmedicated hypertension

\begin{tabular}{lrccc}
\hline & \multicolumn{3}{c}{ Blood pressure } \\
\cline { 2 - 4 } $\begin{array}{l}\text { Abnormal cardiometabolic } \\
\text { risk factors }\end{array}$ & Normal & Prehypertension & Hypertension & Total \\
\cline { 2 - 4 } 0 or 1 & 1518 & 868 & 312 & 2698 \\
2,3 or 4 & 717 & 742 & 375 & 1834 \\
Total & 2235 & 1610 & 687 & 4532 \\
\hline
\end{tabular}

The prevalence ratio for having two or more risk factors (other than high BP) outside of the normal range, in adults with PreHTN vs. subjects with normal BP, was $1.30(1.22,1.39)$.

The prevalence in Jamaica (2012 subjects sampled) was very similar to India, 30\% overall, with a male predominance (35 vs. $25 \%$ in women). ${ }^{24}$ Prevalence in Japan (4706 men and 7342 women) has been reported to be $34.8 \%$ in men and $31.8 \%$ in women, ${ }^{25}$ similar to India and Jamaica. Interestingly the prevalence of PreHTN in Turkey (3015 subjects sampled) was also similar at $38.2 \%$ and doubled the risk for metabolic syndrome, diabetes mellitus and coronary heart disease among women, but only with metabolic syndrome in men. ${ }^{26}$ In China (prospective cohort study with 169871 subjects) hypertensive adults had an increased relative risk for developing CVD (overall, 1.34: women, 1.27; men, 1.42) and mortality ratio (overall, 1.22: women, 1.15 ; men, 1.30). The population-attributable risk for CVD incidence and mortality associated with PreHTN was 10.6 and $7.1 \%$, respectively. The optimal 5-year number-needed-to-treat to prevent a CVD event or death in PreHTN was 53 and 185, respectively. This optimal number-needed-to-treat to prevent a CVD related event was significantly smaller in PreHTN patients with a history of CVD or diabetes ( 34 for incidence and 44 for mortality) compared with those without (115 for incidence and 352 for mortality). ${ }^{27}$ The CVD mortality among the Japanese (12928 subjects followed) was $1.32(0.59-2.94)$, very similar to the Chinese population, with a $7 \%$ population attributable fraction in the 40-64 year age group. ${ }^{28}$

Among the Omani adults with prediabetes (40.9\%), 54.1\% had a history of PreHTN. ${ }^{29}$ These subjects with prediabetes, also in a higher proportion with the male gender and with a higher BMI, were twice as likely to have PreHTN. We have shown that overweight subjects with prediabetes have circadian BP variability abnormalities, ${ }^{10}$ and that obese subjects with prediabetes and with a higher degree of systemic inflammation have both abnormal circadian BP variability and endothelial function abnormalities. ${ }^{30}$ The subjects with abnormalities in both these studies not only had prediabetes, but also had PreHTN. Circadian BP variability abnormalities ${ }^{31,32}$ and endothelial dysfunction ${ }^{33}$ are both early markers for an elevated risk of developing CVD.

The increased risk for cardiovascular morbidity and mortality in PreHTN is not only due to its rapid deterioration into hypertension, ${ }^{19}$ but is also due to the adverse risk profile associated with PreHTN itself. Although the change in the recommended criteria for the 
diagnosis of PreHTN has created some confusion, the results of our study and others suggest that PreHTN maybe a marker for latent CVD. Recent data from various countries show a very similar prevalence of PreHTN in Jamaica, ${ }^{24}$ Japan, ${ }^{25}$ and Turkey ${ }^{26}$ as is being reported here in disease-free adults representative of the US population. The markers for the increased CVD risk in the United States appear to mirror those in China ${ }^{34}$ and Japan: ${ }^{28}$ increase with age, the male gender and BMI (a lower Asian BMI: BMI $>23 \mathrm{~kg} \mathrm{~m}^{-2}$ is considered being overweight in the Japanese population). Enlarged WC, increased TGs and FSG, along with lower HDL-C levels, used for assignment of an adverse cardiometabolic profile in PreHTN, have independent, widespread recognition in the literature for independently elevating CVD risk in otherwise healthy subjects ${ }^{3-10}$ and are also clustered in the diagnostic constructs for the metabolic syndrome. ${ }^{18}$ Recognizing that $\mathrm{BP}$ is a continuous variable, the worldwide prevalence of PreHTN with a tendency to both deteriorate into hypertension and to cluster with other cardiometabolic risk enhancing factors, a concerted effort at intervention is clearly warranted. Because most of these risk-enhancing factors favorably respond to a healthy dietary intake, an increase in exercise, and securing weight loss, in keeping with the JNC 7 guidelines, these need to be the primary measures for intervention.

\section{Conclusion}

These data highlight PreHTN in disease-free healthy adults, a precursor for subsequent hypertension, as an early correlate of an adverse cardiometabolic risk profile. Well-managed clinical interventions designed to prevent the progression from PreHTN to hypertension (based on JNC 7 recommendations) may modulate progression and attenuate risk.

\section{CONFLICT OF INTEREST}

The authors declare no conflict of interest.

1 Lewington S, Clarke R, Qizilbash N, Peto R, Collins R, Prospective Studies Collaboration. Age-specific relevance of usual blood pressure to vascular mortality: a metaanalysis of individual data for one million adults in 61 prospective studies. [Published erratum appears in Lancet 2003; 361:1060]. Lancet 2002; 360: 1903-1913.

2 Whitlock G, Lewington S, Sherliker P, Clarke R, Emberson J, Halsey J, Qizilbash N, Collins R, Peto R. Body-mass index and cause-specific mortality in 900000 adults: collaborative analyses of 57 prospective studies. Prospective Studies Collaboration. Lancet 2009; 373: 1083-1096.

3 Fox CS, Massaro JM, Hoffmann U, Pou KM, Maurovich-Horvat P, Liu CY, Vasan RS, Murabito JM, Meigs JB, Cupples LA, D'Agostino Sr RB, O'Donnell CJ. Abdominal visceral and subcutaneous adipose tissue compartments: association with metabolic risk factors in the Framingham Heart Study. Circulation 2007; 116: 39-48.

4 St-Pierre J, Lemieux I, Perron P, Brisson D, Santuré M, VohI MC, Després JP, Gaudet D. Relation of the 'hypertriglyceridemic waist' phenotype to earlier manifestations of coronary artery disease in patients with glucose intolerance and type 2 diabetes mellitus. Am J Cardiol 2007; 99: 369-373.

5 Haffner SM. Abdominal adiposity and cardiometabolic risk: do we have all the answers? Am J Med 2007; 120 (9 Suppl 1): S10-S16; discussion S16-S17. Review.

6 Patsch JR, Miesenbock G, Hopferwieser T, Muhlberger V, Knapp E, Dunn JK, Gotto Jr AM, Patsch W. Relation of triglyceride metabolism and coronary artery disease: studies in the postprandial state. Arterioscler Thromb 1992; 12: 1336-1345.

7 Castelli WP, Doyle JT, Gordon T, Hames CG, Hjortland MC, Hulley SB, Kagan A, Zukel WJ. HDL cholesterol and other lipids in coronary heart disease. Circulation 1977; 55: 767-772.

8 Manninen V, Tenkanen L, Koskinen P, Huttunen JK, Manttari M, Heinonen OP, Frick $\mathrm{MH}$. Joint effects of serum triglyceride and LDL cholesterol and HDL cholesterol concentrations on coronary heart disease risk in the Helsinki heart study: implications for treatment. Circulation 1992; 85: 37-45.

9 Nielson C, Lange T, Hadjokas N. Blood glucose and coronary artery disease in nondiabetic patients. Diabetes Care 2006; 29: 998-1001.

10 Gupta AK, Greenway FL, Cornelissen G, Pan W, Halberg F. Prediabetes is associated with abnormal circadian blood pressure variability. J Hum Hypertens 2008; 22: $627-633$.
11 Reaven G. The metabolic syndrome or the insulin resistance syndrome? Different names, different concepts, and different goals. Endocrinol Metab Clin North Am 2004; 33: 283-303.

12 Alberti KG, Zimmet PZ. Definition, diagnosis and classification of diabetes mellitus and its complications. Part 1: diagnosis and classification of diabetes mellitus provisional report of a WHO consultation. Diabet Med 1998; 15: 539-553.

13 Expert Panel on Detection, Evaluation, and Treatment of High Blood Cholesterol in Adults. Executive Summary of the Third Report of the National Cholesterol Education Program (NCEP) Expert Panel on Detection, Evaluation, and Treatment of High Blood Cholesterol in Adults (Adult Treatment Panel III). JAMA 2001; 285: 2486-2497.

14 The IDF consensus worldwide definition of the metabolic syndrome. Part 1: Worldwide definition for use in clinical practice. http://www.idf.org. Accessed 28 July 2005.

15 Chobanian AV, Bakris GL, Black HR, Cushman WC, Green LA, Izzi Jr JL, Jones DW, Materson BJ, Oparil S, Wright Jr JT, Rocella EJ, the National High Blood Pressure Education Program Committee. The seventh report of the joint national committee on prevention, detection, evaluation and treatment of high blood pressure. The JNC 7 Report. JAMA 2003; 289: 2560-2572.

16 Reaven GM. The metabolic syndrome: is this diagnosis necessary?. [Published erratum appears in Am J Clin Nutr 2006; 84:1253]. Am J Clin Nutr 2006; 83: 1237-1247.

17 Analytic and Reporting Guidelines: the National Health and Nutrition Examination Survey, NHANES 2005-2006. National Center for Health Statistics, Centers for Disease Control and Prevention: Hyattsville, MD, 2006.

18 Centers for Disease Control and Prevention (CDC). National Center for Health Statistics (NCHS). National Health and Nutrition Examination Survey Questionnaire (or Examination Protocol, or Laboratory Protocol). U.S. Department of Health and Human Services, Centers for Disease Control and Prevention: Hyattsville, MD, 1999-2006. http://www.cdc.gov/nchs/nhanes.htm.

19 Prospective Studies Collaboration. Age-specific relevance of usual blood pressure to vascular mortality: a meta-analysis of individual data for one million adults in 61 prospective studies. Lancet 2002; 360: 1903-1913.

20 Vasan RS, Larson MG, Leip EP, Kannel WB, Levy D. Assessment of frequency of progression to hypertension in non-hypertensive participants in the Framingham Heart Study: a cohort study. Lancet 2001; 358: 1682-1686.

21 De Marco M, de Simone G, Roman MJ, Chinali M, Lee ET, Russell M, Howard BV, Devereux RB. Cardiovascular and metabolic predictors of progression of prehypertension into hypertension. The Strong Heart Study. Hypertension 2009; 54: 974-980.

22 Mainous AG, Everett CJ, Liszka HA, King DE, Egan BM. Prehypertension and mortality in a nationally representative cohort. Am J Cardiology 2004; 94: 1496-1500.

23 Gu Q, Burt VL, Paulose-Ram R, Yoon S, Gillum RF. High blood pressure and cardiovascular disease mortality risk among U.S. adults: the third National Health and Nutrition Examination Survey mortality follow-up study. Ann Epidemiol 2008; 18: 515.

24 Ferguson TS, Younger NO, Tulloch-Reid MK, Wright MB, Ward EM, Ashley DE, Wilks RJ. Prevalence of prehypertension and its relationship to risk factors for cardiovascular disease in Jamaica: analysis from a cross-sectional survey. BMC Cardiovasc Disord 2008; 8: 20.

25 Ishikawa Y, Ishikawa J, Ishikawa S, Kayaba K, Nakamura Y, Shimada K, Kajii E, Pickering TG, Kario K, Jichi Medical School Cohort Investigators Group. Prevalence and determinants of prehypertension in a Japanese general population: the Jichi Medical School Cohort Study. Hypertens Res 2008; 31: 1323-1330.

26 Onat A, Yazici M, Can G, Kaya Z, Bulur S, Hergenç G. Predictive value of prehypertension for metabolic syndrome, diabetes, and coronary heart disease among Turks. Am J Hypertens 2008; 21: 890-895.

27 Gu D, Chen J, Wu X, Duan X, Jones DW, Huang JF, Chen CS, Chen JC, Kelly TN, Whelton PK, He J. Prehypertension and risk of cardiovascular disease in Chinese adults. J Hypertens 2009; 27: 721-729.

28 Hozawa A, Kuriyama S, Kakizaki M, Ohmori-Matsuda K, Ohkubo T, Tsuji I. Attributable risk fraction of prehypertension on cardiovascular disease mortality in the Japanese population: the Ohsaki Study. Am J Hypertens 2009; 22: 267-272.

29 Ganguly SS, AI-Shafaee MA, Bhargava K, Duttagupta KK. Prevalence of prehypertension and associated cardiovascular risk profiles among prediabetic Omani adults. BMC Public Health 2008; 8: 108.

30 Gupta AK, Cornelissen G, Greenway FL, Halberg F, Johnson WD. Increased cardiovascular disease risk in obese: association of altered metabolic profile with abnormal circadian blood pressure variability, and endothelial dysfunction. JASH 2010 (in review)

31 Halberg F, Cornélissen G, Katinas G, Tvildiani L, Gigolashvili M, Janashia K, Toba T, Revilla M, Regal P, Sothern RB, Wendt HW, Wang ZR, Zeman M, Jozsa R, Singh RB, Mitsutake G, Chibisov SM, Lee J, Holley D, Holte JE, Sonkowsky RP, Schwartzkopff O, Delmore P, Otsuka K, Bakken EE, Czaplicki J, International BIOCOS Group. Chronobiology's progress: season's appreciations 2004-2005. Time, frequency, phase, variable, individual, age- and site-specific chronomics. J Appl Biomed 2006; 4: 1-38.

32 Halberg F, Cornélissen G, Katinas G, Tvildiani L, Gigolashvili M, Janashia K, Toba T, Revilla M, Regal P, Sothern RB, Wendt HW, Wang ZR, Zeman M, Jozsa R, Singh RB, Mitsutake G, Chibisov SM, Lee J, Holley D, Holte JE, Sonkowsky RP, Schwartzkopff O, Delmore P, Otsuka K, Bakken EE, Czaplicki J, International BIOCOS Group. Chronobiology's progress: part II, chronomics for an immediately applicable biomedicine. J Appl Biomed 2006; 4: 73-86.

33 Corrado E, Rizzo M, Coppola G, Muratori I, Carella M, Novo S. Endothelial dysfunction and carotid lesions are strong predictors of clinical events in patients with early stages of atherosclerosis: a 24-month follow-up study. Coron Artery Dis 2008; 19: 139-144.

34 Yu D, Huang J, Hu D, Chen J, Cao J, Li J, Gu D. Association between prehypertension and clustering of cardiovascular disease risk factors among Chinese adults. J Cardiovasc Pharmacol 2009; 53: 388-400. 